Corresponding Author:

Raluca-loana Dascălu

Received 22 June 2020

Accepted 3 September 2020

Published

30 September 2020

Production and Hosting by

Knowledge E

(c) Raluca-loana Dascălu et al. This article is distributed under the terms of the

Creative Commons

Attribution License, which

permits unrestricted use and redistribution provided that

the original author and

source are credited.

Editor-in-Chief:

Prof. Mohammad A. M. Ibnouf
Research Article

\section{The Role of PET-CT in Gastric Cancer - A Narrative Review}

\section{Raluca-loana Dascălu ${ }^{1}$, Dan Nicolae Păduraru ${ }^{1,2}$, Alexandra Bolocan ${ }^{1,2}$, Daniel Ion ${ }^{1,2}$, and Octavian Andronic ${ }^{1,2}$}

${ }^{1}$ The University of Medicine and Pharmacy Carol Davila, Bucharest, Romania

${ }^{2}$ University Emergency Hospital of Bucharest, Romania

\section{Abstract}

Background: Gastric cancer is one of the most common and leading causes of cancer death worldwide, with an increasing risk and prevalence. Although the usage of 18-FDG PET-CT in gastric cancer evaluation remains a matter of debate and is not consistently recommended by international guidelines, our descriptive review aims to highlight its actual role in the diagnostic accuracy, staging, therapeutic management, and relapse monitoring of this malignancy.

Methods: The current research was conducted using scholarly databases including PubMed, Scopus, and Google Scholar by searching useful science journals, references, and abstracts on the topic. The keywords used were "gastric cancer" AND "PET-CT". Results: 18-FDG PET-CT remains a promising method with increasing clinical utility not only across a wide variety of malignancies, but also among gastric cancer patients.

Conclusions: We are certain that with further improvements, this technique could improve the diagnosis and evaluation of gastric cancer, and make it more approachable and accurate.

Keywords: gastric cancer (GC), PET-CT, 18-FDG PET-CT, 18F-FDG uptake, ceCT (contrast-enhanced CT)

\section{Introduction}

Gastric cancer is one of the most common and leading causes of cancer death worldwide, with an increasing risk and prevalence. When talking about gastric cancer, we usually refer to adenocarcinomas, whereas this histopathological type represents about $90 \%$ of the cases. There are also other less-common gastric malignancies, such as lymphomas, gastrointestinal stromal tumors (GIST), neuroendocrine tumors, or metastases located at this level.

Geographical variations seem to be marked in the incidence of this malignancy, S OPEN ACCESS with the highest rates reported in East Asia, South America, and Eastern Europe and 
the lowest rates in the United States and Western Europe [1]. Moreover, studies have shown that the incidence has increased among elderly patients with peaks in the seventh decade, especially in men, who are more commonly affected than women, with the male incidence rates being twice as that of females. Also, there is an inverse proportional link between gastric cancer incidence and social class, with lower rates in professional groups and higher rates in unskilled laborers [2]. However, in Western countries, survival rates seem to increase progressively, and in Japan, due to the routine screening programs and the focused management of prognosis-related factors, it seems to be the highest [3].

It is well-known that there are plenty of factors involved in the etiology of gastric cancer, such as Helicobacter pylori infection, smoking and alcohol, dietary factors, atrophic gastritis, partial gastrectomy, Epstein-Barr virus, or genetic predisposition.

Based on the anatomical location, tumors can either be proximal (cardiac and esogastric junction) or distal (basal, bodily or antral); the incidence of the proximal tumors seems to be increasing. Microscopically, there are several histological classifications for gastric adenocarcinoma, and the most widely used classification was proposed by Lauren, dividing gastric cancer into diffuse and intestinal types [2].

When it comes to 18-FDG PET uptake, it might be difficult to differentiate the normal uptake from pathological. Many benign lesions like polyps, gastritis, or even normal gastric mucosa could show moderate-to-intense 18F-FDG uptake, hence 18F-FDG might not be a cancer-specific agent [5]. In the nondistended stomach, 18-FDG PET may normally present diffusely increased uptake, as well as in the gastroesophageal junction. In this case, it is also likely to be physiological due to normal muscular contraction of the lower esophageal sphincter, when there are no CT abnormalities. A similar situation can be found in gastroesophageal reflux disease [6].

Moreover, several studies have shown that there is a considerable interdependence between 18-FDG PET uptake and patient survival and that standardized uptake values (SUVs) are important in follow-up of patients and estimating the prognosis. So, it seems that unlike patients with low SUVs, those with high tumor SUVs had lower prognosis with lower rates of survival [7]. Also, a strong connection can be noticed between SUV and the primary size of tumors [7], whereas, if tumors exceed more than $3.5 \mathrm{~cm}$ and have a deeper invasion, detection rates also increase. Additionally, it looks like that the intestinal type of gastric adenocarcinoma is associated with a higher mean SUV (7.7), while in mucinous and signet ring cell tumors, there is a modest FDG activity (SUV mean, 4.2). The explanation for this could be the lower expression of GLUT-1 transporters on 
the cell membrane surface, an increased amount of intracellular mucin in mucinous and signet ring cell tumors, or the decreased cellularity [6].

An accurate evaluation and early diagnosis are important in deciding not only regarding the prognosis, but also the treatment. For the patients who present with advanced gastric cancer (AGC), some efficient techniques are in demand for the accuracy of staging and quantitative evaluation, so as to enhance an appropriate selection of treatments. It is of great importance for patient management and surgical planning of the lymph node involvement, distant metastasis and an accurate staging, including the full disclosure of the local invasion extent [4]. Moreover, the results of PET imaging proved to have a powerful impact on patient management, either by avoiding previously planned therapeutic procedures or by using previously unexpected therapeutic procedures [30]. Considering the great importance of the therapy outcomes in the management of the patients, with personalized treatment and guiding future therapy improvements, it appears that PET-CT technology could be effective in monitoring tumor response to treatments and might show prognostic value with the potential to change therapeutic strategies [3].

It seems that over the years, the clinical evaluation of gastric cancer has strongly improved with the conventional tools like computed tomography (CT), magnetic resonance imaging (MRI), endoscopic ultrasound (EUS), PET-CT and laparoscopic staging, although they all have some technical limitations [3]. Using 18F-fluorodeoxyglucose (FDG), positron emission tomography (PET) turned out to have significant advantages in therapeutic evaluation, staging, metastases, and recurrence in plenty of malignancies by providing metabolic information about tumors [4]. Basically, in contrast with other imaging tools, such as $C T$ and MRI, which reflect primarily anatomical structure, PET reflects biological functions in tissue and allows noninvasive imaging of physiological parameters within the body, such as metabolic activity and perfusion, using tracers labeled with positron-emitting radioisotopes. In PET, the spatial distribution and concentration of administered positron-emitting compounds are used to generate cross-sectional images through the body that reflect pathological states or elucidate physiological processes, leading to applications in clinical practice as well as in research. The principal clinical uses of PET in gastroenterology are in the area of oncology [2].

\section{Materials and Methods}

In order to approach and maybe give a better understanding of how gastric malignancies could be present and recognized on 18-FDG PET-CT, we analyzed the studies of patients 
performed and described in literature articles. Our research was conducted on PubMed, Scopus, and Google Scholar databases comprising useful science journals, references, and abstracts on the topic of our interest, and using the following keywords: "gastric cancer" AND "PET-CT". The results were filtered by year (published in the last 10 years), language (English), and article type (original article and review).

\section{Results}

Due to the unclear impact of 18-FDG PET-CT on gastric cancer staging, the primary lesion $(T)$ staging, the lymph node staging $(N)$, and the distant metastases $(M)$ staging, also the involvement of 18-FDG PET-CT in response evaluation and relapse monitoring, we followed the results and discussions described in the different studies.

It is commonly believed that 18-FDG PET-CT improves TNM staging. Taking into consideration the primary lesion staging, different factors may influence the diagnostic accuracy, such as the histological type of the tumor, its size or location, as well as the physiological FDG uptake by the gastric wall [4]. It looks like, among studies, when using 18F-FDG-Pet alone for the detection of primary lesion, the sensitivity rate ranges between 58 and 94\% (median 81.5\%) and the specificity, however, ranges between 78 and $100 \%$ (median 100\%). Also, when it comes to early gastric cancer (EGC), it has been noticed that the detection rate might be even lower: in EGC, only the intestinal type could be detected with 18F-FDG-PET [8]. Although there is no agreement on its benefit, the National Comprehensive Cancer Network (NCCN) recommends the use of 18F-FDG PETCT in the preoperative staging of gastric cancer. Besides, several studies investigated its role in the primary staging of gastric cancer in comparison with ceCT (contrastenhanced $\mathrm{CT}$ ) as routine staging method. For instance, in a study which investigated a group of 31 patients ( $24 \mathrm{M}$; $7 \mathrm{~F}$; mean age: $58.9 \pm 12.6$ years) for primary staging of gastric cancer during one year (between June 2011 and June 2012), primary tumor was detected in 20 patients by 18 F-FDG PET-CT and in 23 patients with ceCT [9]. Also, while in the first clinical studies, the accuracy, sensitivity, and specificity of 18F-FDG PET-CT for detecting primary tumor has been reported as 93\%, 100\%, and 95\%, respectively [10], further studies showed a sensitivity as low as $60 \%$. On the other hand, in advancedstage gastric cancer $(A G C)$, regarding the detection of primary tumor, a sensitivity of PET-CT as 93\% and ceCT as 90\% was reported by Young KE et al. [11]; also, Shimizu $\mathrm{K}$ et al. discovered that mucosal cancers have a lower detection rate than submucosal ones (16.6 vs 68.8\%) [12]. It appears that, even though recent studies suggest that there might be an evolving role for the diagnosis of primary gastric tumors, literature suggests 
the potential utility of performing later time-point images to improve the detectability of gastric cancer [13]. Therefore, FDG-PET provides little information concerning the layer of the gastric wall involved and the invasion of adjacent organs.

When it comes to gastric lymph nodes, an effective classification belongs to the Japanese Association for Gastric Cancer, according to which there are 16 perigastric ganglion groups, divided in four drainage stations from N1 to N4, within the location of the primary tumor. This grouping system is the result of studies of lymphatic flow in different tumor locations, along with survival associated with metastases at each nodal station. Thus, the first station ("proximal" ganglion station - N1) consists of the first six groups (left and right paracardial LN, LN along the left, the right and the short gastric vessels, $L N$ along the left and the right gastroepiploic vessels, suprapyloric $L N$ and infrapyloric LN); the second station ("distal" ganglion station - N2) consists of: group 7 - LN along the left gastric artery, group 8 - LN along the common hepatic artery, group 9 - LN along the celiac artery, group 10 - LN along the splenic hilum, and group 11 - LN along the splenic artery. The rest of the groups (12-16) constitutes the metastatic N3 and N4 stations: group 12 - LN in the hepatoduodenal ligament, group 13 - retropancreatic LN, group 14 - LN along the mesenteric artery, group 15 - LN along the middle colic artery, and group 16 - satellite LN of the aorta and inferior vena cava. Depending on the gastrectomy performed being partial or total, D1 dissection is defined as a dissection of all the first group nodes and some of the station 7 nodes. D2 dissection is defined as dissection of all the first and the second group nodes, while D3 is defined as dissection of all the groups. Since in AGC, all patients undergo D1 dissection anyway, it seems that detecting $\mathrm{N} 1$ disease status might not influence the surgical management of patients with gastric adenocarcinoma [14]. So that, a greater clinical impact could determine the $\mathrm{N} 2$ or N3 disease status, considering that the presence of N2 disease might increase the extent of lymph node dissection from D1 to D2 or greater [15]. It is considered necessary to lift a minimum of 15 nodes together with the resection piece.

Comparing with EUS, MRI, or CT, no significant diagnostic difference was reported for $\mathrm{N}$ staging in gastric malignancies. Literature has pointed out major limitations of CT in detecting cancerous involvement of normal-sized lymph nodes; also, it cannot differentiate between reactive hyperplasia and metastatic enlargement. However, other individual studies claimed that FDG-PET or PET-CT was less sensitive, but more specific, contrasting with commonly used CT and MRI [17, 18]. Furthermore, in a series of 59 patients confirmed with regional lymph node metastases, while the accuracy and sensitivity of PET-CT was found to be inferior to $\mathrm{CeCT}$, the specificity and positive predictive value for PET-CT seemed to be considerably higher than for CT [11]. Both 
the 18F-FDG-PET and CT have reduced sensitivity in detecting N2 and N3 lymph node stations, but even so, the specificity of 18F-FDG-PET ranges from 91 to $100 \%$ (median: 96\%) for N1 and N2 stations [7].

There are plenty of factors influencing or compromising the sensitivity of FDG-PET for $\mathrm{N}$ staging, such as the histological type of the primary tumor, the size of metastatic lymph nodes, or the high physiological uptake background from the normal gastric wall. For instance, while some cell types might be detected with moderate sensitivity (30-71\%), others, like signet ring cell carcinoma, reveal the lowest sensitivity (15\%) [7]. While the current 18F-FDG-PET scanners have 4-10 mm spatial resolution, perigastric N1 lymph nodes or metastatic lymph nodes smaller than 3-4 mm are beyond the detection capacity of most PET scanners. In order to represent local metastasis, regional lymph nodes should meet certain criteria: round shape, exhibiting central necrosis with marked or heterogeneous enhancement, should be solitary or separate nodes of $8 \mathrm{~mm}$ or greater in the long axis diameter. However, literature suggests that $55 \%$ of the metastatic lymph nodes usually have a diameter of $5 \mathrm{~mm}$ or less [16]. Additionally, the nearby primary tumor could generate a radioactive volume effect, so that the discrimination of some small lymph nodes could be even more difficult [4]. Besides, a link has been shown between the SUV $V_{\max }$ of the lymph nodes and the SUV $\mathrm{V}_{\max }$ of the primary tumor and one report pointed out that in patients without FDG-avid primary tumors, $60-70 \%$ of lymph node metastases could not be detected [11]. Another study revealed that a poor prognosis in this malignancy is significantly associated with a high nodal SUVmax and, so as to predict survival in gastric cancer, metabolic information of metastatic lymph nodes represented by nodal SUVmax could be more important than primary tumor [5].

Taking all the discussions into account, we can affirm that FDG- PET or PET-CT usually proves a higher specificity than other commonly used imaging techniques in the $\mathrm{N}$ staging of gastric cancer. Furthermore, owing to this high specificity, PET-CT has valuable potential for determining lymphadenectomy or reducing futile laparotomies [6].

It is commonly known that the most frequently targeted sites of distant metastases from gastric adenocarcinomas are within the abdominal cavity (including the peritoneal surfaces, liver, adrenals, kidneys, ovaries, and spleen), in the left supraclavicular lymph nodes, and in the bones. Frequently, distant metastases are detected by using contrast $\mathrm{CT}$, however, PET-CT might be more useful in detecting distant sites of solid organ metastases because it seems an excellent adjunct modality to detect these anatomically small but potentially metabolically active focuses of metastatic disease [19]. Thus, recent studies showed that FDG-PET-CT imaging was able to detect solid organ metastasis (lungs, liver, bone, or adrenal gland) with high sensitivity (95.2\%) and specificity (100\%) 
[20]. Regarding bone metastases, whole-body bone scanning is the most preferred way to evaluate the status, so as, one study revealed the comparison between the value of FDG-PET and whole-body bone scintigraphy for the determination of bone metastasis in gastric cancer patients. The authors of the study concluded that FDG-PET was superior for the detection of synchronous bone metastasis, even if the two modalities had a similar sensitivity and accuracy [21].

When compared with CT, FDG-PET usually shows a lower sensitivity especially for determining peritoneal seeding, maybe because of the small and diffuse growing patterns or the diffuse histological type of gastric cancer. Therefore, many studies propose CT for diagnosing peritoneal metastasis because of its high quality [22] However, even if for identifying peritoneal metastases of gastric cancer it might not increase benefit to high-quality contrast CT, using FDG-PET-CT in addition to CT, EUS or laparoscopy prove to have some advantages both therapeutically and economically speaking; hence, unecessary gastrectomy could be avoided in almost $10 \%$ of patients and more than $\$ 10,000$ could be saved per patient [23].

Despite its limitations, the literature has proven that preoperative 18F-FDG-PET-CT could provide meaningful information and emerge as an effective instrument not only for therapeutic evaluation, but also for prognosis and recurrence after surgical resection in patients with gastric cancer. Although collective data does not appear to fully agree, preoperative FDG uptake has been established as an autonomous and considerable prognostic factor after curative gastrectomy [24]. Conventional imaging techniques might be noninvasive and the cheapest of the available methods, but they present limitations regarding the accuracy of recurrence assessment and they have limited role in differentiating post-surgical changes from local tumor recurrence [25]. In one study evaluating 130 patients, 91 of them had recurrent disease, while the remaining 39 were negative for recurrence. When comparing the final diagnosis with PET-CT findings, among 91 patients with true recurrence, 83 showed positive and 8 showed negative FDG uptakes. The remaining 39 patients who did not have true recurrence showed positive FDG uptakes in 15 and negative in 24 patients. Therefore, the sensitivity turned out to be $91.2 \%$, specificity $61.5 \%$, and accuracy of PET-CT $82.3 \%$. Taking the results into account, the study suggests that PET-CT might be greatly effective in detecting or confirming recurrence once a patient with gastric cancer is clinically or radiologically suspicious of recurrence during follow-up [25].

Another study evaluated the correlation between SUVmax and cancer recurrence: Lee and colleagues [26] noticed that on 18F-FDG-PET, a SUVmax equal or higher than 8.2, represents a poor prognostic factor associated with an increasing risk of 
cancer recurrence after curative surgical resection for gastric cancer patients who did not receive any neoadjuvant therapy. In addition, a significantly lower incidence of recurrence and better recurrence-free survival was seen after the surgery in patients with lower preoperative FDG uptake in the GC $[4,24]$.

Also, there are debates focused on the comparison between $\mathrm{ceCT}$, the most frequently used imaging technique for gastric cancer, and FDG PET-CT, concerning aftersurgery surveillance. While some studies suggest that these two might share a similar performance in detecting post-surgery recurrence [27], others reveal that FDG PET-CT might be superior. Hence, analyzing both the sensitivity and specificity of FDG PET-CT in determining gastric cancer recurrence after surgical removal, two recent meta-analyses discovered they were $78-86 \%, 82-88 \%$, respectively $[28,29]$.

Further, with regards to the treatment planning and response to therapy, a majority of patients still relapse following resection; surgical removal with lymph node dissection is currently the only treatment modality that is potentially curative, multidisciplinary treatment planning being mandatory. Even so, in addition to the surgical removal for gastric cancer patients, preoperative chemotherapy or radiotherapy have been gaining a great interest and recently has been used in order to improve the relapse-free and overall survival [4].

Even if conflicting reports claimed that the survival rate did not reveal a considerable difference between patients with tumor FDG uptakes and those without, evidence has shown that FDG-PET-CT could be an efficient and non-invasive tool concerning therapeutic evaluation. For instance, lower preoperative FDG uptake could predict the curability of the tumor. On the contrary, a positive FDG uptake in local lymph nodes with a higher FDG uptake in the primary tumor have been strongly correlated with noncurative resection, suggesting that these patients should volunteer for neoadjuvant $\mathrm{CHT}$ [24]. Furthermore, after beginning the treatment, changes in FDG uptake related to final outcome were observed. Therefore, literature revealed that while metabolic responders proved better prognosis, neoadjuvant therapies might be ineffective in metabolic nonresponders and in patients with reduced FDG uptake [4].

\section{Discussions}

Taking everything into account, the main limitations of FDG-PET and PET-CT in diagnosing and evaluating this malignancy arise from the spatial resolution of PET, the histological diversity in gastric cancer and, from the physiological properties of the stomach. When talking about spatial resolution, in the future, it could be improved by 
optimizing the design of the camera [4]. Depending on the tumor being intestinal or non-intestinal, considering that many FDG non-avid histological types strongly reduce the sensitivity of FDG-PET and PET-CT to detect gastric cancer, there are currently under evaluation new tracers such as FLT-PET and some metabolic agents as alternatives $[4,7]$. As for the physiological properties of the stomach, pharmaceutical interventions, which include muscle relaxants, proton pump inhibitors, and gastric distention by different kind of methods are under debate [4].

Furthermore, studies have reported a limited significance of PET in the detection of peritoneal carcinomatosis with a low sensitivity (range: 9-50\%; median: 32.5\%), but relatively higher specificity (63-99\%; median: 88.5\%) [7]. This reduced sensitivity could be justified by a small number of tumor cells in pleural, ascites, and bone metastases, the small size of peritoneal lesions $(<5 \mathrm{~mm})$, or the fibrosis associated [31]. $\mathrm{N}$ staging in gastric cancer is yet not adequate under current circumstances. Nevertheless, despite the limitations, 18F-FDG-PET provides useful information on estimating the prognosis and the aggressiveness of tumor.

Overall, combining the higher sensitivity of CT with higher specificity of PET could be even more beneficial for improving the accuracy of many diseases, including gastric cancer, rather than the use of imaging alone [7]. As future prospects, the clinical usefulness of PET-CT could further increase with application of more effectively techniques. Hopefully, the new generation of multimodality imaging equipment, such as PET-MR or PET-CT-MR, will ensure considerable benefits in the diagnosis and evaluation of gastric cancer and many different malignancies [4].

\section{Conclusion}

In the light of the aforementioned discussion, although it seems to be a matter of controversy, the value of 18F-FDG PET-CT remains promising. Over the years literature revealed its lower sensitivity due to physical FDG-uptake or involuntary movements of the gastric wall, when compared with other malignancies or other imaging techniques like ceCT. The limitations of 18F-FDG PET-CT have been proven not only in the early stage and signet ring cell carcinomas, but also in the detection of lymph node metastasis. On the other hand, data suggest that PET-CT could be used in demonstrating occult metastatic disease or for patients in whom metastatic cancer is not evident. Furthermore, other studies support the utility of PET-CT in gastric cancer staging, particularly in describing distant or lymphatic metastases beyond compartment I or II. Additionally, some data supports its potential to change therapeutic strategies by higher 
precision in preoperative staging preventing worthless surgery and higher sensitivity in detecting recurrence in postoperative follow-up of patients; also, needless prolonged chemotherapy and the related toxicity could be prevented with the anticipation of early response to therapy for 18F-FDG-avid tumors. Thus, 18-FDG PET-CT remains a promising method with increasing clinical utility not only across a wide variety of malignancies, but also in gastric cancer patients. Therefore, with further improvements, this technique could enhance the diagnosis and evaluation of gastric cancer and make it more approachable.

\section{Declaration section}

\section{Acknowledgements}

None

\section{Competing Interests}

None

\section{Availability of Data and Material}

Open data

\section{Funding}

None

\section{References}

[1] Waddell, T., Verheij, M., Allum, W., et al. (2013). Gastric cancert: ESMO-ESSOESTRO clinical practice guidelines for diagnosis, treatment and follow-up. Annals of Oncology, vol. 24, no. 6.

[2] Yamada, T., Alpers, D. H., Kalloo, A. N., et al. (2009). Textbook of Gastroenterology ( $5^{\text {th }}$ ed.), vol. 1-2, Blackwell Publishing Ltd, USA

[3] De Raffele, E., Mirarchi, M., Cuicchi, D., et al. (2017). Evolving role of FDG$\mathrm{PET} / \mathrm{CT}$ in prognostic evaluation of resectable gastric cancer. World Journal of 
Gastroenterology, vol. 23, no. 38, pp. 6923-6926.

[4] Wu, C. X. and Zhu, Z. H. (2014). Diagnosis and evaluation of gastric cancer by positron emission tomography. World Journal of Gastroenterology, vol. 20, no. 16, pp. 4574-4585.

[5] Song, B. I., Kim, H. W., Won, K. S., et al. (2015). Preoperative standardized uptake value of metastatic lymph nodes measured by 18F-FDG PET/CT improves the prediction of prognosis in gastric cancer. Medicine, vol. 94, no. 26., pp 1-8

[6] Malibari, N., Hickeson, M., and Lisbona R. (2015). PET/computed tomography in the diagnosis and staging of gastric cancers. PET Clinics, vol. 10, no. 3, pp. 311-326.

[7] Atay-Rosenthal S., Wahl, R. L., and Fishman, E. K. (2012). PET/CT findings in gastric cancer: potential advantages and current limitations. Imaging in Medicine, vol. 4, no.

2. Retrieved from: https://www.openaccessjournals.com/articles/petct-findingsin-gastric-cancer-potential-advantages-and-current-limitations.html?fbclid= IwAR1fmJh5yTSIzly2PpLkcckmVZnOSpLu4za9zeLJ866agHGZvRLvsPmFj5। [accessed on December 16, 2019].

[8] Mukai, K., Ishida, Y., Okajima, K., et al. (2006). Usefulness of preoperative FDG-PET for detection of gastric cancer. Gastric Cancer, vol. 9, no. 3, pp. 192-196.

[9] Filik, M., Kir, K. M., Aksel, B., et al. (2015). The role of 18F-FDG PET/CT in the Primary staging of gastric cancer. Molecular Imaging and Radionuclide Therapy, vol. 24, no. 1, pp. 15-20.

[10] Yeung, H. W. D., Macapinlac, H., Karpeh, M., et al. Accuracy of FDG-PET in gastric cancer. preliminary experience. Molecular Imaging and Biology, vol. 1, no. 4, pp. 213-221.

[11] Kim, E. Y., Lee, W. J., Choi, D., et al. (2011). The value of PET/CT for preoperative staging of advanced gastric cancer: comparison with contrast-enhanced CT. European Journal of Radiology, vol. 79, no. 2, pp. 183-188.

[12] Shimizu, K., Ito, K., Matsunaga, N., et al. (2005). Diagnosis of gastric cancer with MDCT using the water-filling method and multiplanar reconstruction: CT-histologic correlation. American Journal of Roentgenology, vol. 185, no. 5, pp. 1152-1158.

[13] Perlaza, P., Ortín, J., Pagès, M., et al. (2018). Should 18F-FDG PET/CT Be routinely performed in the clinical staging of locally advanced gastric adenocarcinoma? Clinical Nuclear Medicine, vol. 43, no. 6, pp. 402-410.

[14] Yun, M., Lim, J. S., Noh, S. H., et al. (2005). Lymph node staging of gastric cancer using 18F-FDG PET: a comparison study with CT. Journal of Nuclear Medicine, vol. 46 , no. 10 , pp. 1582-1588. 
[15] Tamura, S., Takeno, A., and Miki, H. (2011). Lymph node dissection in curative gastrectomy for advanced gastric cancer. International Journal of Surgical Oncology, vol. 2011, no. 1 pp. $1-8$.

[16] Kwee, R. M. and Kwee, T. C. (2009). Imaging in assessing lymph node status in gastric cancer. Gastric Cancer, vol. 12, no. 1. pp. 6-22.

[17] Seevaratnam, R., Cardoso, R., McGregor, C., et al. (2012). How useful is preoperative imaging for tumor, node, metastasis (TNM) staging of gastric cancer? A metaanalysis. Gastric Cancer, vol. 15, no.1, pp. S3-S18.

[18] Ha, T. K., Choi, Y. Y., Song, S. Y., et al. (2011). F18-fluorodeoxyglucose-positron emission tomography and computed tomography is not accurate in preoperative staging of gastric cancer. Journal of Korean Surgical Society, vol. 81, no. 2, pp. 104-110.

[19] Hopkins, S. and Yang, G. Y. (2011). FDG PET imaging in the staging and management of gastric cancer. Journal of Gastrointestinal Oncology, vol. 2, no. 1, pp. 39-44.

[20] Chung, H. W., Lee, E. J., Cho, Y.-H., et al. (2010). High FDG uptake in PET/CT predicts worse prognosis in patients with metastatic gastric adenocarcinoma. Journal of Cancer Research and Clinical Oncology, vol. 136, no. 12, pp. 1929-1935.

[21] Ma, D. W., Kim, J.-H., Jeon, T. J., et al. (2013). 18F-fluorodeoxyglucose positron emission tomography-computed tomography for the evaluation of bone metastasis in patients with gastric cancer. Digestive and Liver Disease, vol. 45, no. 9, pp. 769775.

[22] Wang, Z. and Chen, J. Q. (2011). Imaging in assessing hepatic and peritoneal metastases of gastric cancer: a systematic review. BMC Gastroenterology, vol. 11, no,1, p. 19

[23] Smyth, E., Schöder, H., Strong, V. E., et al. (2012). A prospective evaluation of the utility of 2-deoxy-2-[18F] fluoro-D-glucose positron emission tomography and computed tomography in staging locally advanced gastric cancer. Cancer, vol. 118, no. 22. pp. 5481-5488.

[24] Yun, M. (2014). Imaging of gastric cancer metabolism using 18 F-FDG PET/CT. Journal of Gastric Cancer, vol. 14, no. 1, pp. 1-6.

[25] Cayvarlı, H., Bekiş, R., Akman, T., et al. (2014). The role of 18F-FDG PET/CT in the evaluation of gastric cancer recurrence. Molecular Imaging and Radionuclide Therapy, vol. 23, no. 3, pp. 76-83.

[26] Lee, J. W., Lee, S. M., Lee, M. S., et al. (2012). Role of 18F-FDG PET/CT in the prediction of gastric cancer recurrence after curative surgical resection. European Journal of Nuclear Medicine and Molecular Imaging, vol. 39, no. 9, pp. 1425-1434. 
[27] Sim, S. H., Yu, J. K., Oh D.-Y., et al. (2009). The role of PET/CT in detection of gastric cancer recurrence. BMC Cancer, vol. 9, p. 73.

[28] Wu, L. M., Hu, J. N., Hua, J., et al. (2012). 18F-fluorodeoxyglucose positron emission tomography to evaluate recurrent gastric cancer: a systematic review and metaanalysis. European Journal of Gastroenterology \& Hepatology, vol. 27, no. 3, pp. 472-480.

[29] Zou, H. and Zhao, Y. (2013). 18FDG PET-CT for detecting gastric cancer recurrence after surgical resection: a meta-analysis. Surgical Oncology, vol. 22, no. 3, pp. 162166.

[30] Bilici, A., et al. (2011). The role of 18F-FDG PET/CT in the assessment of suspected recurrent gastric cancer after initial surgical resection: can the results of FDG PET/CT influence patients' treatment decision making? European Journal of Nuclear Medicine and Molecular Imaging, vol. 38, no. 1, pp. 64-73.

[31] Dassen, A. E., Lips, D. J., Hoekstra, C. J., et al. (2009). FDG-PET has no definite role in preoperative imaging in gastric cancer. European Journal of Surgical Oncology, vol. 35 , no. 5 , pp. 449-455. 